07.2

\title{
Влияние единичного граничного ловушечного заряда на подпороговый ток стока FinFET-транзистора с различной формой канала
}

\section{(C) A.E. Абдикаримов}

Ургенчский государственный университет, Ургенч, Узбекистан

E-mail: abdukarimov.azamat@rambler.ru

Поступило в Редакцию 3 октября 2019г.

В окончательной редакции 2 марта 2020 г.

Принято к публикации 2 марта 2020г.

\begin{abstract}
Моделируется влияние формы канала вертикального полевого транзистора с изолированным затвором на амплитуду случайного телеграфного шума, индуцированного единичным зарядом, встроенным на граничных ловушках. Рассматриваются транзисторы с прямоугольным и трапециевидным поперечным сечением канала. Показано, что при встраивании единичного граничного заряда в потолке канала в подпороговой области напряжений на затворе амплитуда шума для транзистора с трапециевидным поперечным сечением меньше, чем для транзистора с прямоугольным сечением. Однако при встраивании единичного заряда в середине боковой поверхности канала амплитуда случайного телеграфного шума существенно больше для трапециевидного сечения.
\end{abstract}

Ключевые слова: случайный телеграфный шум, FinFET-транзистор, граничный ловушечный заряд, плотность тока стока.

DOI: 10.21883/PJTF.2020.10.49430.18060

С уменьшением размеров МОП-транзисторов (МОП - металл-оксид-полупроводник) влияние флуктуации различных параметров на его характеристики усиливается. В частности, встраивание локального оксидного заряда может привести к изменению различных характеристик $[1,2]$, а единичная граничная заряженная ловушка может изменить ток стока и вызвать случайные телеграфные шумы (СТШ) в наноразмерных МОПтранзисторах [3]. СТШ привлекает очень большое внимание исследователей по многим причинам, среди которых можно отметить исключительную важность устойчивости к СТШ работоспособности ячеек памяти, основанных на МОП-структурах [4-6], а также то, что изменения порогового напряжения, вызванные СТШ, могут быть значительно выше, чем индуцированные случайной флуктуацией примеси [7]. Кроме того, наряду с другими методами исследования свойств различных слоев $[8,9]$ исследования СТШ дают возможность характеризовать ловушки в оксидном слое МОП-транзисторов [10].

СТШ исследовались в основном в планарных МОП-транзисторах, и только в последние годы усилился интерес к исследованиям СТШ в вертикальных двух-, трехзатворных (FinFET) транзисторах, транзисторах с полностью обедненным каналом (FDSOI), а также беспереходных (Junctionless MOSFET) полевых транзистоpax [7,11]. В этих работах в основном сравнивается влияние СТШ на характеристики транзисторов, изготовленных по различным технологиям. Опубликованы работы, в которых рассматриваются зависимости амплитуды СТШ сигнала от толщины оксидного слоя, ширины канала, соотношения толщины канала к ширине, поло- жения единичного граничного ловушечного заряда на боковой поверхности [12,13]. Исследовалась взаимосвязь вариации таких внутренних параметров, как случайная флуктуация концентрации примеси, линейная краевая шероховатость, с амплитудой СТШ [14]. Моделировалась взаимосвязь СТШ с количеством и положением единичной ловушки вдоль канала полевого транзистоpa [15]. Однако практически нет работ, посвященных исследованию амплитуды СТШ в вертикальных транзисторах, в частности FinFET-транзисторах с различной формой канала, хотя при формировании наноразмерных транзисторов в технологических процессах могут иметь место флуктуации формы канала.

В настоящей работе проводится моделирование СТШ сигнала, генерированного единичным зарядом, захваченным на граничной ловушке, расположенной как в центре потолка, так и в боковой поверхности канала FinFET-транзистора, основанного на технологии „кремний на изоляторе“ (КНИ), с различной формой канала. Рассматривались каналы с формой в поперечном сечении в виде прямоугольника и трапеции (рис. $1, a, b)$.

Для проведения 3D-моделирования использовалась программа Advanced TCAD Sentaurus. При моделировании в используемой диффузионно-дрейфовой модели учитывались зависимость подвижности от уровня легирования и насыщение скорости носителей при высоких полях, а также влияние нормальной составляющей поля на ток стока. Нанометровые масштабы транзистора требуют учета квантовых эффектов. Для учета этих эффектов использовалась квантовая корректировка по градиенту плотности (density gradient quantum 


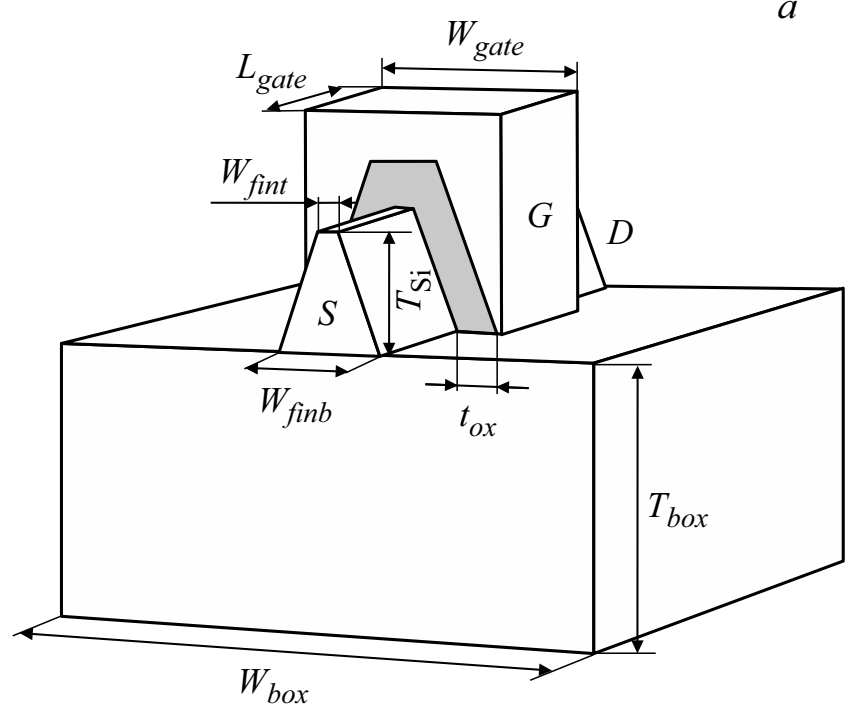

Рис. 1. Структура моделируемого КНИ FinFET-транзистора с трапециевидным $(a)$ и прямоугольным $(b)$ сечением.

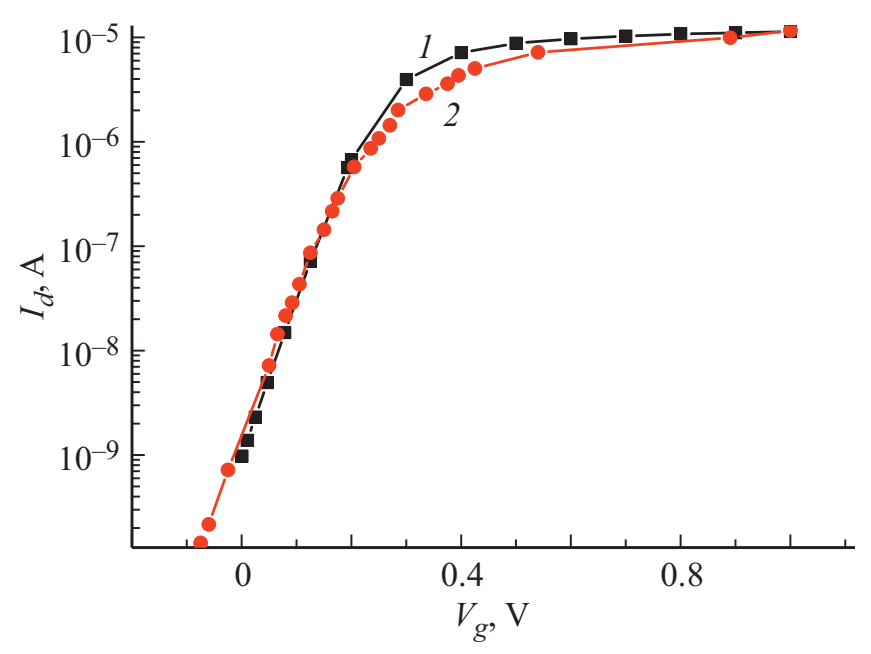

Рис. 2. Калибровка моделируемого транзистора (1) экспериментальными результатами работы [18] (2) при $L_{\text {gate }}=25 \mathrm{~nm}$ и $V_{d s}=50 \mathrm{mV}$.

correction) [16,17]. Используемая модель калибровалась по экспериментальным результатам работы [18] (рис. 2). Встроенный единичный граничный заряд моделировался однородно заряженной круглой поверхностью с радиусом $0.5 \mathrm{~nm}$ и поверхностной плотностью $1.27 \cdot 10^{14} \mathrm{~cm}^{-2}$, вписанной на границе. При моделировании длина поликремниевого затвора $(G)$ принята равной $L_{\text {gate }}=10 \mathrm{~nm}$. В качестве подзатворного оксидного слоя взят $\mathrm{HfO}_{2}$ с эквивалентной толщиной $t_{o x}=0.35 \mathrm{~nm}$. Форма канала определялась по ширине его потолка $W_{f i n t}$, которая для рассматриваемого трапецеидального сечения равна $5 \mathrm{~nm}$. Канал транзистора легирован бором с концентрацией $10^{15} \mathrm{~cm}^{-3}$, имеет ширину у основания $W_{\text {finb }}=10 \mathrm{~nm}$ и высоту $T_{\mathrm{Si}}=10 \mathrm{~nm}$. Стоковая и истоковая области легированы фосфором с гауссовым профилем и максимальной концентрацией $10^{20} \mathrm{~cm}^{-3}$. Ширина и толщина скрытого оксидного слоя равны соответственно $W_{b o x}=100 \mathrm{~nm}$ и $T_{b o x}=100 \mathrm{~nm}$.

Результаты представлены в виде зависимости амплитуды СТШ сигнала от приращения напряжения на затворе $V_{g}$ над пороговым $V_{t h}\left(V_{g}-V_{t h}\right)$ для двух рассматриваемых форм канала (рис. 3 ). Из результатов видно, что при пороговых и меньших напряжениях на затворе амплитуда СТШ сигнала, вызванного единичным зарядом, захваченным на граничной ловушке в середине потолка (рис. 3, кривые 1,2 ) и боковой поверхности (рис. 3 , кривые 3,4 ), заметно больше по сравнению с надпороговым значением. При этом как в случае прямоугольного, так и в случае трапециевидного сечения амплитуда СТШ сигнала выше при захвате единичного заряда на боковой поверхности. Это связано с тем, что максимальная плотность тока протекает в нижней половине канала, ближе ко дну канала (рис. $3, b)$, что более удалено от середины потолка, чем от середины боковой поверхности. В то же время амплитуда СТШ, вызванная граничным единичным зарядом, встроенным на середине боковой поверхности, больше для трапециевидного, чем для прямоугольного сечения. Это можно объяснить приближением ловушечного заряда к области максимальной плотности тока стока при трапециевидном сечении канала (вставка на рис. $3, a$ ). Сравнительно большая плотность тока стока при прямоугольном сечении канала (рис. $3, b$ ) индуцирует больший сигнал СТШ, чем при трапециевидном сечении, для случая захвата единичного заряда на потолке канала.

Таким образом, результаты моделирования показывают, что форма канала в КНИ FinFET-транзисторе заметно влияет на амплитуду СТШ сигнала в подпороговой области передаточной характеристики транзи- 

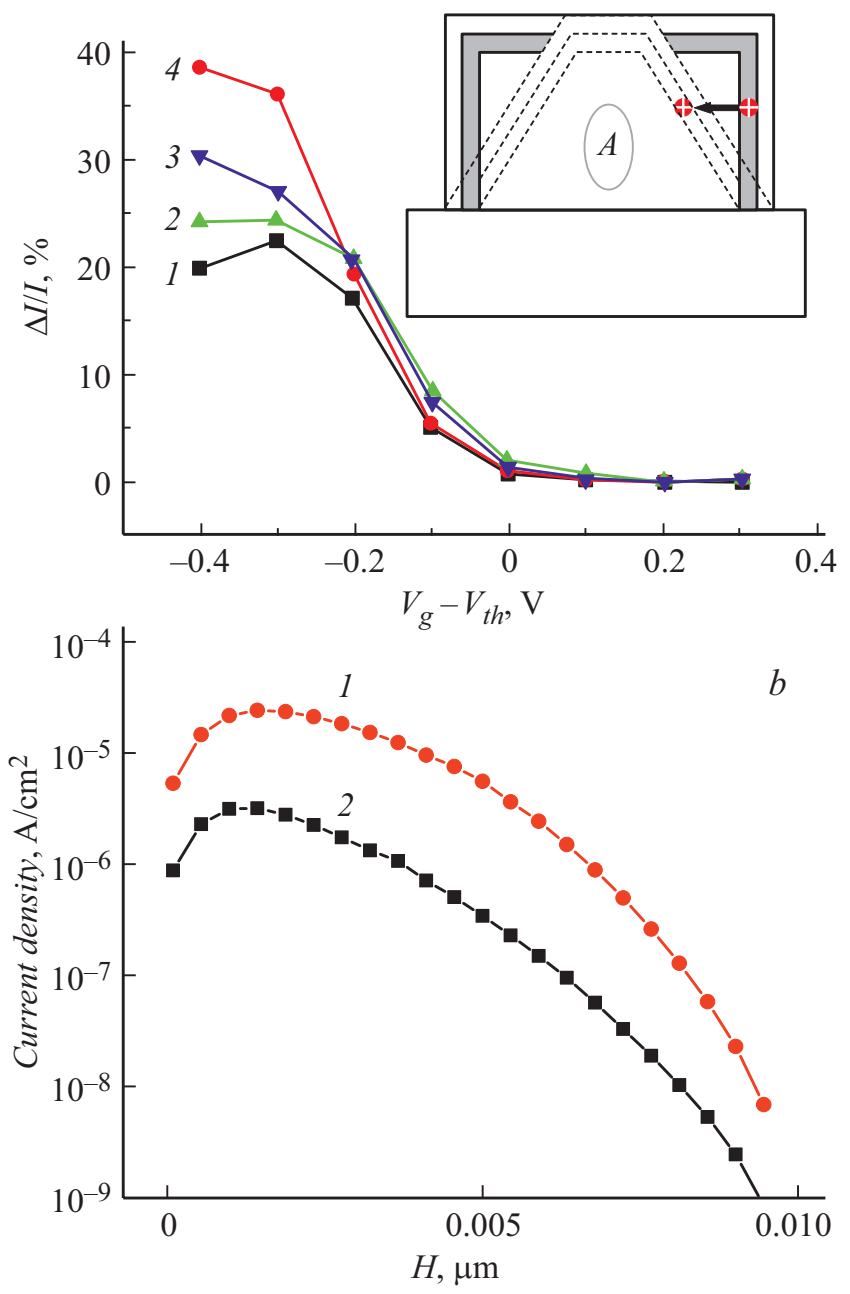

Pис. 3. $a-$ зависимость амплитуды СТШ от приращения напряжения на затворе над пороговым для транзистора с трапециевидным $(1,4)$ и прямоугольным $(2,3)$ сечением канала при встраивании единичного граничного заряда на середине потолка $(1,2)$ и середине боковой поверхности $(3,4)$. На вставке показано смещение положения единичного бокового заряда к области максимальной плотности тока $A$ в канале при изменении сечения канала с прямоугольного на трапециевидное. $b-$ распределение плотности тока стока в середине канала по высоте $H$ для прямоугольного $(1)$ и трапециевидного (2) сечений канала.

стора. В случае встраивания единичного ловушечного граничного заряда на потолке канала амплитуда СТШ меньше для транзистора с каналом трапециевидного сечения, чем для прямоугольного поперечного сечения. И это связано с большей величиной плотности тока стока в канале в случае прямоугольного сечения. При встраивании единичного граничного заряда на боковой поверхности канала амплитуда СТШ больше для случая трапециевидного сечения канала, что связано с уменьшением расстояния между ловушечным зарядом и областью максимальной плотности тока стока в канале.

\section{Конфликт интересов}

Автор заявляет, что у него нет конфликта интересов.

\section{Список литературы}

[1] Атамуратова 3.А., Юсупов А., Халикбердиев Б.О., Атамуратов А.Э. // ЖТФ. 2019. Т. 89. В. 7. С. 1067-1070.

[2] Atamuratov A.E., Atamuratova Z.A., Yusupov A., Ghani A. // Results Phys. 2018. V. 11. P. 656-658.

[3] Campbell J.P., Yul L.C., Cheung K.P., Qin J., Suehle J.S., Oates A., Sheng $K$. Large random telegraph noise in subthreshold operation of nano-scale nMOSFETs // Proc. IEEE Int. Conf. IC Design and Technology (ICICDT). IEEE, 2009. P. 17-20.

[4] Toh S.O., King Liu T.-J., Nikolié B. Impact of random telegraph signaling noise on SRAM stability // 2011 Symp. on VLSI Technology. Digest of technical papers. IEEE, 2011. P. 204-205.

[5] Yamaoka M., Miki H., Bansal A., Wu S., Frank D.J., Leobandung E., Torii K. Evaluation methodology for random telegraph noise effects in SRAM arrays // IEDM Tech. Dig. IEEE, 2011. P. 745-748.

[6] Zou J., Wang R., Gong N., Huang R., Xu X., Ou J., Liu C., Wang J., Liu J., Wu J., Yu S., Ren P., Wu H., Lee S.-W., Wang $Y$. New insights into AC RTN in scaled high-k/metalgate MOSFETs under digital circuits operations // 2012 Symp. on VLSI Technology. Digest of technical papers. IEEE, 2012. P. $139-140$

[7] Fan M.-L., Yang S.-Y., Hu V.P.-H., Chen Y.-N., Su P., Chuang C.-T. // Microelectron. Reliability. 2014. V. 54. P. 698 711.

[8] Dittmar K., Triyoso D.H., Erben E., Metzger J., Binder R., Brongersma H.H., Weisheit M., Engelmann H.-J. // Surf. Interface Anal. 2017. V. 49. P. 1175-1186. https://doi.org/10.1002/sia.6312

[9] Кутлиев У.О., Каримов М.К., Отабоев М.У. // Физика и химия обраб. материалов. 2019. № 1. С. 5-10.

[10] Shin H., Oh B. Characterization of oxide traps by RTN measurement in MOSFETs and memory devices // 17th IEEE Int. Symp. on the physical and failure analysis of integrated circuits. IEEE, 2010. P. 1-5.

DOI: 10.1109/IPFA.2010.5531998

[11] Gerrer L., Amoroso S.M., Hussin R., Asenov A. // Microelectron. Reliability. 2014. V. 54. P. 1749-1752.

[12] Fan M.-L., Hu V.P.-H., Chen Y.-N., Su P., Chuang C.-T. // IEEE Trans. Electron. Dev. 2012. V. 59. N 8. P. 2227-2234.

[13] Lu B.K.Y., Fan M.-L., Su P. Impact of aspect ratio on the subthreshold RTN amplitude of multi-gate MOSFETs // Proc. of the Int. Conf. on solid state devices and materials. Nagoya, 2011. P. 84-85. DOI: 10.7567/SSDM.2011.P-3-1

[14] Wang X., Brown A.R., Cheng B., Asenov A. RTS amplitude distribution in $20 \mathrm{~nm}$ SOI FinFETs subject to statistical variability // Int. Conf. on simulation of semiconductor processes and devices (SISPAD). Denver, 2012. P. 296-299.

[15] Both T.H., Firpo Furtado G., Wirth G.I. // Microelectron. Reliability. 2018. V. 80. P. 278-283.

[16] Garcia-Loureiro A.J., Seoane N., Aldegunde M., Valin R., Asenov A., Martinez A., Kalna K. // IEEE Trans. ComputerAided Design Integr. Circuits Syst. 2011. V. 30. N 6. P. 841851 . 
[17] Asenov A., Brown A., Watling J. // Solid-State Electron. 2003. V. 47. P. 1141-1145.

[18] Basker V.S., Standaert T., Kawasaki H., Yeh C.-C., Maitra K., Yamashita T., Faltermeier J., Adhikari H., Jagannathan H., Wang J., Sunamura H., Kanakasabapathy S., Schmitz S., Cummings J., Inada A., Lin C.-H., Kulkarni P., Zhu Y., Kuss J., Yamamoto T., Kumar A., Wahl J., Yagishita A., Edge L.F., Kim R.H., Mclellan E., Holmes S.J., Johnson R.C., Levin T., Demarest J., Hane M., Takayanagi M., Colburn M., Paruchuri V.K., Miller R.J., Bu H., Doris B., McHerron D., Leobandung E., O'Neill $J$. A $0.063 \mu \mathrm{m}^{2}$ FinFET SRAM cell demonstration with conventional lithography using a novel integration scheme with aggressively scaled fin and gate pitch // Symp. on VLSI Technology. Digest of technical papers. IEEE, 2010. P. 19-20. 